OPEN ACCESS

Edited by:

Jienan Pan,

Henan Polytechnic University, China

Reviewed by:

Greeshma Gadikota,

Cornell University, United States

Niu Qinghe,

Shijiazhuang Tiedao University, China

Zhenzhi Wang,

China University of Mining and

Technology, China

*Correspondence:

Yixin Zhao

zhaoyx@cumtb.edu.cn

Specialty section: This article was submitted to

Economic Geology,

a section of the journal

Frontiers in Earth Science

Received: 29 September 2021

Accepted: 29 November 2021

Published: 15 December 2021

Citation:

Liu T, Zhao Y and Noraei Danesh N (2021) The Characteristics of Closed Pores in Coals With Different Ranks.

Front. Earth Sci. 9:785913.

doi: 10.3389/feart.2021.785913

\section{The Characteristics of Closed Pores in Coals With Different Ranks}

\author{
Tong Liu ${ }^{1}$, Yixin Zhao ${ }^{1,2 *}$ and Nima Noraei Danesh ${ }^{1,2}$ \\ ${ }^{1}$ School of Energy and Mining Engineering, China University of Mining and Technology (Beijing), Beijing, China, ${ }^{2}$ Beijing Key \\ Laboratory for Precise Mining of Intergrown Energy and Resources, China University of Mining and Technology (Beijing), Beijing, \\ China
}

The closed pores in coal seams influence the storage of coalbed methane. The investigation of closed pores characteristics for coals is of great significance in improving the production of coalbed methane and revealing the mechanism of coal and gas outburst. However, due to limitations in analytical techniques, the characteristics and evolution mechanism of closed pores in coals with different ranks are not sufficiently understood. In this paper, eight coal samples with different ranks were collected and characterized by small-angle $\mathrm{X}$-ray scattering (SAXS) and low-temperature nitrogen adsorption (LTNA). The open and closed pores of coals with various ranks were studied, and the mechanism for evolution of closed pores during coalification was proposed. The results show that among eight coal samples with different ranks, the closed porosity of low-metamorphic coals is relatively lower, the closed porosity of medium-metamorphic coals is in the middle, and the closed porosity of high-metamorphic coals is relatively higher. The change in closed porosity for coals with different ranks may be related to varieties of the molecular structure of coals. The low-metamorphic coals have more disordered arrangement of molecular structure and easily form connected pores. Therefore, the closed porosity in low-metamorphic coals is low. The aromatization of medium-metamorphic coals turns aliphatic chains into closed aromatic rings, and the closed porosity of these coals also increases. When coals reach a high degree of metamorphism, polycondensation compacts the coal macromolecular structure, providing for easy formation of closed pores between aromatic condensed rings, so the closed porosity is obviously increased in high-metamorphic coals. This study has dual significance in advancing the understanding of open and closed pores in coals and the mechanism of coal and gas outburst.

Keywords: coal, small-angle X-ray scattering, low-temperature nitrogen adsorption, closed pore, different ranks

\section{INTRODUCTION}

Coal is a sedimentary organic rock evolved from the burial and accumulation of ancient plant remains going through a long period of geological, physical and chemical processes (Dai et al., 2020). With the geological evolution of coal, the molecular structure of coal change regularly (Liu et al., 2018). With the cleavage and expulsion of aliphatic side chains and functional groups in coal, a large amounts of methane and other gases are produced (Levine, 1993). Much of methane exists in coal seams, which is a serious hazard hindering the safe operation of coal mines (Flores, 1998). 
A great amount of gas can be stored in coal seams, and this is closely related to the large amounts of nanopores in coals (Yao et al., 2008). These pores provide large specific surface areas for adsorption of methane molecules, thus ensuring the gas adsorption capacity in coal seams (Hu et al., 2020). Pores in coal include open pores and closed pores (Alexeev et al., 1999; Nie et al., 2015). The pores in coal are divided into passing, interconnected, dead end and closed pores. According to the pores connectivity, the former three types are considered as open pores, which can be detected by injecting probe fluids methods. Coalification causes the nanopores of coal to change regularly, and the mechanism for pore evolution is essentially controlled by evolution of molecular structure in coal (Li et al., 2020). The evolution of pore structure must be accompanied by evolution of the characteristics of open and closed pores during coalification (Wang et al., 2020). Furthermore, coalification also determines the gas content generated during evolution of the coal seam (Bustin and Clarkson, 1998). Therefore, it is possible that some generated gas may be trapped in the closed pores of coal (Chen et al., 2018).

Coal and gas outburst has plagued coal mine safety for a long time, and its scientific mechanism is still of interest in the field of coal mining safety research (Wang et al., 2019). Mining activities of coal seam disrupted its original equilibrium state. The enclosed gas originally stored in the closed pores of the coal seam may be connected with the outside environment. A great amount of gas is instantly desorbed, leading to mining disasters such as coal and gas outburst (Niu et al., 2017). Therefore, coal and gas outburst may be related to the gas existing in the closed pores of the coal seam. Furthermore, the total gas content of an experimental coal sample generally includes lost gas, desorbed gas and residual gas (Chen et al., 2019). The residual gas may initially be sealed in closed pores in coal. The exploitation of gas trapped in closed pores is challenging, so closed pores affect the overall exploitation efficiency of coalbed methane. Ignoring the influence of closed pores in coal may lead to overestimation of coalbed methane productivity (Chen et al., 2018). Therefore, studying the characteristics of closed pores in coal has immense theoretical and practical importance in preventing coal and gas outburst disaster, recognizing the mechanism for storage of coalbed methane and improving accuracy in evaluation of coalbed gas content.

Since it is difficult for probes to contact closed pores directly, direct detection of closed pores in coal is not easy (Mou et al., 2021). There are many techniques for investigating the pore structure of coal. The common methodologies include mercury porosimetry, low-temperature nitrogen adsorption (LTNA), carbon dioxide adsorption, etc (Han et al., 2020). However, these injecting fluids methods can only detect the open pores, which are difficult to detect closed pores in coal. (Mou et al., 2021). Other techniques used to characterize microscopic morphology mainly include scanning electron microscopy (SEM) (Li et al., 2019), transmission electron microscopy (TEM) (Zhao et al., 2014), high-resolution transmission electron microscopy (HRTEM) (Wang et al., 2021) and micro-computed tomography (CT) (Li et al., 2017). However, they are limited to observing the local pore structure of samples and cannot effectively evaluate the overall pore characteristics of coal seams. In short, research on closed pores in coal is limited.

While Small-angle X-ray scattering is an advanced nondestructive testing method. It can detect nano-scale pore characteristics depending on the electron density difference between coal matrix and pores, including open and closed pores (Okolo et al., 2015). Radlinski et al. (2004) used smallangle X-ray scattering (SAXS) and small-angle neutron scattering (SANS) to investigate the pore structure of seven coal samples and analyzed their pore parameters. Pan et al. (2016) used small angle X-ray scattering (SAXS) and low-temperature nitrogen adsorption (LTNA) to study three tectonic coals with similar metamorphic degrees and analyzed the influence of tectonic deformation on the properties of closed pores in coal.

In this study, the SAXS technique was used to investigate total pore characteristics (including both open and closed pores) of coal with different ranks. Additionally, LTNA experiments were carried out with coal samples to measure the open pore characteristics of each sample. Generally, the effective testing range of LTNA is $1.8-300 \mathrm{~nm}$, while the pore diameter of sample characterized were approximately $2-65 \mathrm{~nm}$ by SAXS in this study. There is overlap of testing range between two methods, so the characteristics of open and closed pores within the range of 2-65 $\mathrm{nm}$ can be determined for coal samples. With the combined application of the two methods, the characteristics of open and closed pores for coal samples were effectively measured, and mechanism for evolution of open and closed pores during the coalification process was analyzed. A scheme for effectively measuring the characteristics of closed pores in coal was put forward in this paper. It is of substantial significance to promote research on the characteristics of open and closed pores in coal and to understand the mechanism of coal and gas outburst.

\section{EXPERIMENTAL WORK}

\section{Sample Preparation}

Eight coal samples were collected in representative coalfields in China, their ranks ranging from low metamorphic lignite to high metamorphic anthracite. Samples GS (lignite) and SGT (bituminous C) were taken from Pingzhuang Coalfield and Dongsheng Coalfield in Inner Mongolia Province respectively. Sample XZY (bituminous C) was obtained from Datong Coalfield in Shanxi Province. These coal seams belong to the Jurassic coals. Sample TS (bituminous B) was collected from Kailuan coalfield in Hebei Province. Sample ZB (bituminous B) was obtained from the Huoxi coalfield in Shanxi Province. Samples ZC (bituminous A), YW (bituminous A) and NY (anthracite) were obtained from the Qinshui coalfield in Shanxi Province. These five coal samples belong to the Carboniferous-Permian coals. Fresh coal samples were gathered carefully from mining working faces. Then, samples were vacuum-sealed and immediately brought to test in the laboratory.

Block samples were broken into smaller piece samples with a hammer in the laboratory. A sample of each coal with a diameter larger than $1 \mathrm{~cm}$ was selected and polished into a slice sample 
TABLE 1 | The maximum vitrinite reflectance, proximate analyses and coal rank of coal samples.

\begin{tabular}{|c|c|c|c|c|c|c|c|c|}
\hline \multirow[t]{2}{*}{ Sample ID } & \multirow[t]{2}{*}{ Sample location } & \multirow{2}{*}{$\begin{array}{c}\text { Coal seam } \\
\text { NO. }\end{array}$} & \multirow[t]{2}{*}{$R_{\text {o,max }}(\%)$} & \multicolumn{4}{|c|}{ Proximate analyses(\%) } & \multirow[t]{2}{*}{ Coal rank } \\
\hline & & & & $\mathbf{M}_{\mathrm{ad}}$ & $\mathbf{V}_{\text {daf }}$ & $\mathbf{A}_{\mathrm{d}}$ & $\mathbf{F C}_{\mathrm{ad}}$ & \\
\hline GS & Gushan & $6 \#$ & 0.44 & 11.57 & 40.56 & 9.09 & 47.16 & Lignite \\
\hline SGT & Shigetai & 3\# & 0.62 & 9.22 & 36.65 & 6.25 & 53.55 & Bituminous $\mathrm{C}$ \\
\hline$X Z Y$ & Xinzhouyao & $11 \#$ & 0.81 & 1.56 & 34.54 & 7.77 & 59.35 & Bituminous $\mathrm{C}$ \\
\hline TS & Tangshan & 9\# & 1.12 & 1.6 & 32.83 & 8.57 & 60.34 & Bituminous B \\
\hline ZB & Zhengbang & $11 \#$ & 1.33 & 0.98 & 27.01 & 6.33 & 67.66 & Bituminous B \\
\hline ZC & Zhangcun & $3 \#$ & 1.72 & 1.02 & 18.36 & 9.49 & 73.06 & Bituminous $\mathrm{A}$ \\
\hline YW & Yuwu & 3\# & 2.07 & 0.54 & 13.36 & 9.35 & 78.07 & Bituminous A \\
\hline NY & Nanyang & $3 \#$ & 4.25 & 1.23 & 9.63 & 11.43 & 78.93 & Anthracite \\
\hline
\end{tabular}

$R_{o, \text { max }}$, mean maximum vitrinite reflectance; $d$, dry basis; ad, air-dry basis; daf, dry ash-free basis; $M_{\text {ad, }}$ moisture on air- $d r y$ basis; $V_{\text {daf, }}$ volatile matter on dry ash-free basis; $A_{d}$, ash on dry basis; $F C_{a d}$, fixed carbon on air-dry basis.

TABLE 2 | The ultimate analyses and true density of coal samples.

\begin{tabular}{lcccccc}
\hline Sample ID & \multicolumn{4}{c}{ Ultimate analyses(\%) } & True density $\left(\mathbf{g} / \mathbf{c m}^{\mathbf{3}}\right)$ \\
\cline { 2 - 6 } & $\mathbf{C}_{\text {daf }}$ & $\mathbf{H}_{\text {daf }}$ & $\mathbf{O}_{\text {daf }}$ & $\mathbf{N}_{\text {daf }}$ & $\mathbf{S}_{\mathbf{t} \text {,daf }}$ & \\
\hline GS & 74.19 & 5.44 & 17.45 & 1.26 & 1.66 & 1.37 \\
SGT & 79.25 & 4.37 & 14.63 & 1.07 & 0.68 & 1.4 \\
XZY & 83.56 & 4.65 & 10.36 & 0.97 & 0.46 & 1.38 \\
TS & 85.39 & 4.87 & 7.94 & 1.32 & 0.48 & 1.34 \\
ZB & 86.21 & 4.12 & 6.88 & 1.45 & 1.34 & 1.32 \\
ZC & 87.62 & 4.03 & 6.37 & 1.47 & 0.51 & 1.38 \\
YW & 89.83 & 3.92 & 4.69 & 1.18 & 0.38 & 1.44 \\
NY & 91.25 & 2.66 & 3.64 & 1.18 & 1.27 & 1.61
\end{tabular}

daf, dry ash-free basis; O content is calculated by difference.

with $\sim 1 \mathrm{~cm}$ diameter and $\sim 1 \mathrm{~mm}$ thickness with sandpaper. The prepared slice samples were used for SAXS experimental study. Furthermore, crushed coal samples with small particles were collected and ground. After pulverization, particle samples of 60-80 mesh were selected for low-temperature nitrogen adsorption experiments and subsequent analyses.

Based on Chinese national standards GB/T 30,732-2014, the proximate analyses of coal samples were performed by using the automatic analyzer. Vitrinite reflectance measurements were conducted on polished coal samples employing a Leitz MPV-3 photometer microscope in accordance with Chinese national standards GB/T 6948-2008. The proximate analyse and maximum vitrinite reflectance results are listed in Table $\mathbf{1}$. The ultimate analyses of coal samples were performed according to Chinese national standards GB/T 31391-2015. The automatic true density analyzer was used to test the true relative density of coal samples. The results are presented in Table 2 .

\section{Small-Angle X-Ray Scattering Experiment}

The SAXS experiments were conducted at the 1W2A line station of Beijing Synchrotron Radiation Facility (BSRF) (Li et al., 2014). The incident monochromatic $\mathrm{X}$-ray beam was provided with energy fixed at $8.052 \mathrm{keV}$ and its wavelength is fixed at $0.154 \mathrm{~nm}$. The focused X-ray spot size is about $1.4 \mathrm{~mm}$ (horizontal) $\times$ $0.2 \mathrm{~mm}$ (vertical). The detectable region of scattering vector $(q=$ $4 \pi \sin \theta / \lambda)$ is mainly determined by the length of the SAXS camera for detector. In this experiment, the distance between sample and detector was $1,570 \mathrm{~mm}$, and the measured scattering vector $(q)$ ranges from 0.097 to $3.339 \mathrm{~nm}^{-1}$.

The sample holder with a cavity of $10 \mathrm{~mm}$ in diameter and $1 \mathrm{~mm}$ thickness was used to place test sample. The prepared circular slice samples were put into the cavity and fixed for SAXS experiment. This experiment was conducted in the air environment, and the air background was subtracted in subsequent data processing. To calculate pore structural parameters, the absolute scattering intensity is necessary (Xie et al., 2018). In this study, the absolute scattering intensity was calibrated by using the glassy carbon SRM 3600 (Xie et al., 2018).

The mass of each sample was weighed prior to SAXS experiment in order to compare the differences in pore structure for samples with a same mass standard. The results are presented in Table 3 .

The porosity of porous materials can be calculated from the invariant $Q_{i n v}$ as Eq. 1 (Radlinski, 2006; Xie et al., 2018):

$$
Q_{i n v}=\int_{0}^{\infty} q^{2}\left(\frac{\partial \Sigma}{\partial \Omega}\right)_{s}(q) d q=2 \pi^{2}\left(\Delta \rho_{e} \cdot r_{e}\right)^{2} P(1-P)
$$

where, $q$ is the scattering vector $\left(\mathrm{nm}^{-1}\right),\left(\frac{\partial \Sigma}{\partial \Omega}\right)_{s}(q)$ is the absolute scattering intensity $\left(\mathrm{cm}^{-1}\right), \Delta \rho_{e}$ is the electron density difference between solid matrix and pore in the material $\left(\mathrm{e} / \AA^{3}\right), r_{e}$ is the classical electron radius which is equal to $2.8179 \times 10^{-13} \mathrm{~cm}$, and $P$ is the porosity (\%).

Coal is considered to be a two-phase polydisperse system composed of coal matrix and pores (Radlinski et al., 2004). The electron density of pores in coal approximates zero. Therefore, the electron density difference $\Delta \rho_{e}$ between the coal matrix and the pores is about the electron density of coal matrix.

The electron density of coal matrix can be calculated by the true relative density and ultimate analyses results as Eq. 2 (Radlinski, 2006):

$$
\rho_{e}=\frac{\rho N_{A} \sum \alpha_{i} Z_{i}}{\sum \alpha_{i} M_{i}}
$$

where, $\rho$ is true relative density, $N_{A}=6.022 \times 10^{23}$ is Avogadro's number, $i$ represents a kind of element, $\alpha_{i}$ is the content with percent of element $i, Z_{i}$ is the number of electrons of element $i, M_{i}$ is the molecular weight of element $i$. 
TABLE 3 | The mass of different experimental samples.

\begin{tabular}{lcccccccc}
\hline Sample ID & GS & SGT & XZY & TS & ZB & ZC & YW & NY \\
\hline Mass $(g)$ & 0.0627 & 0.0714 & 0.0822 & 0.0831 & 0.0858 & 0.0872 & 0.0935 & 0.1062 \\
\hline
\end{tabular}

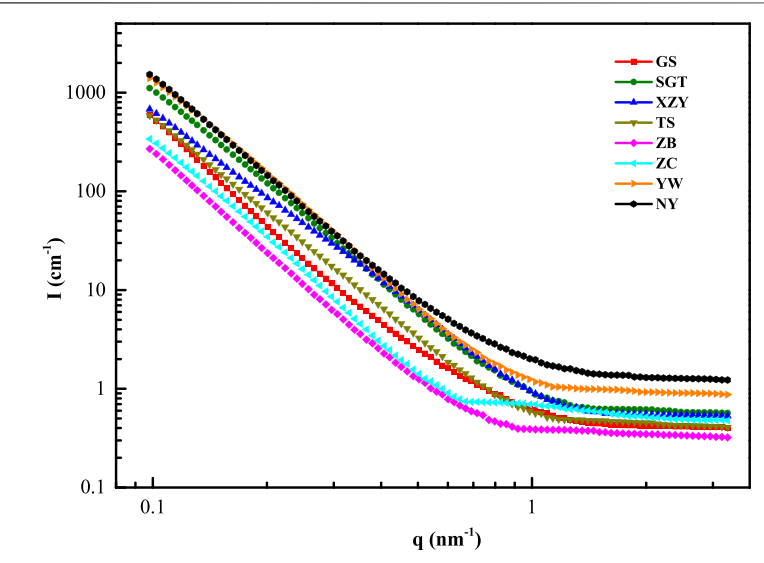

FIGURE 1 | Scattering curves of eight coal samples with different ranks.

The real space size of a scatterer is shown in Eq. 3 ( $\mathrm{Li}$ et al., 2018):

$$
\frac{2 \pi}{q_{\max }} \leq d \leq \frac{2 \pi}{q_{\min }}
$$

where, $q$ is the scattering intensity $\left(\mathrm{nm}^{-1}\right)$ and $d$ is the pore diameter $(\mathrm{nm})$. The measured scattering vector $(q)$ ranges from 0.097 to $3.339 \mathrm{~nm}^{-1}$ in this experiment, so the pore diameter of each sample characterized were approximately 2-65 nm. Detailed information on processing SAXS experimental data was provided in our previous publication (Zhao et al., 2020).

\section{Low-Temperature Nitrogen Adsorption Experiment}

Low-temperature nitrogen adsorption experiments were performed on an ASAP 2020Plus surface area and porosity analyzer (Micromeritics, United States). According to China's national standard GB/T 21650.2-2008, the test was conducted by using the static volumetric method. Samples prepared from 60 to 80 mesh particles were selected for the LTNA experiment.

Prior to analysis, $1.0 \mathrm{~g}$ of each coal sample was degassed under vacuum at $110^{\circ} \mathrm{C}$ for approximately $10 \mathrm{~h}$, then the degassed sample was weighed again. Afterward, the test tube was connected to the experimental instrument for analysis. An appropriate amount of high-purity liquid nitrogen (purity >99.999\%) was put into the Dewar flask to provide the lowtemperature of liquid nitrogen $(77 \mathrm{~K})$ for this experiment.

Both adsorption and desorption isotherms were tested at the relative pressure $\left(\mathrm{P} / \mathrm{P}_{0}\right)$ in the range of $0.01-0.995(\mathrm{P}$ is the equilibrium pressure and $\mathrm{P}_{0}$ is the saturated vapor pressure at the temperature of liquid nitrogen, approximately $0.1013 \mathrm{MPa}$ ).

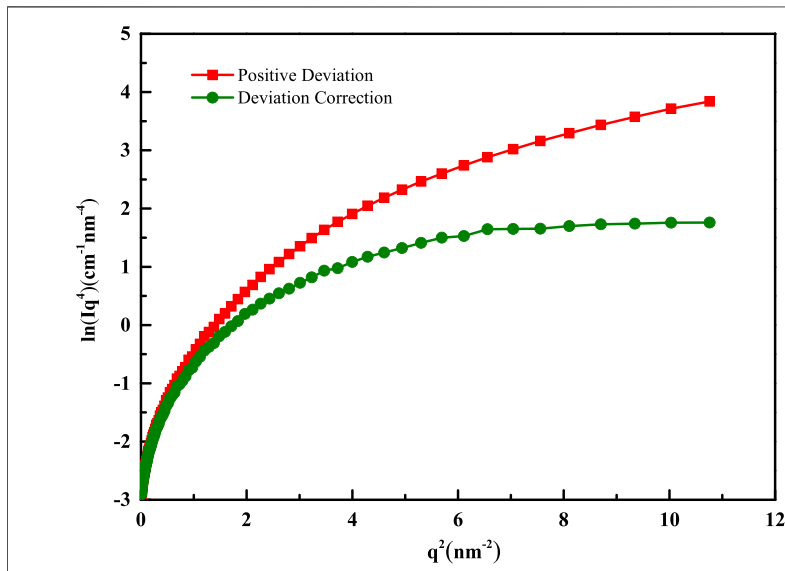

FIGURE 2 | Porod's curve deviation correction for sample (GS sample as an example).

The equilibrium state during entire experimental process was controlled automatically. Then, the isotherms of adsorption and desorption were obtained. Finally, the pore structure parameters of samples were acquired by the instrumental software.

\section{RESULTS AND DISCUSSION}

\section{Small-Angle X-Ray Scattering Results}

The scattering curves for eight coal samples with different ranks are presented in Figure 1. The overall trends are similar in the scattering curves for samples, and the scattering intensity $I$ of the samples decrease with scattering vector $q$ increasing. Over the entire scattering vector range of $0.097-3.339 \mathrm{~nm}^{-1}$, sample NY and sample YW exhibit high scattering intensity $I$, while sample $\mathrm{ZB}$ has the lowest scattering intensity $I$, and the scattering intensity $I$ of other samples are between it of samples NY and $\mathrm{ZB}$. For most samples, when the scattering vector $q$ is $1 \mathrm{~nm}^{-1}$, the scattering intensity $I$ decreases to a low value. When the scattering vector $q$ is larger than $1 \mathrm{~nm}^{-1}$, the scattering intensity $I$ basically remains stable or decreased slightly. The scattering curves for different samples reflect the differences in nanopores structure, which may be mainly related to the different ranks of coal samples (Zhao et al., 2014).

When processing SAXS data, it is necessary to calibrate the initial experimental data based on Porod's theory. In this study, the original Porod's curve of each sample shows obvious positive deviation effects. This is due to the existence of non-uniform regions of nano-scale electron density in the sample (Li, 2013). The electron density of coal matrix fluctuates slightly because of heterogeneity of coal matrix and presence of mineral matter 
TABLE 4 | Pore volume per unit mass of each coal sample.

\begin{tabular}{lcc}
\hline Sample ID & $\boldsymbol{R}_{\text {o, } \mathbf{m a x}}(\mathbf{\%})$ & Pore volume $\left(\mathbf{m m}^{\mathbf{3}} \mathbf{/ g}\right)$ \\
\hline GS & 0.44 & 17.587 \\
SGT & 0.62 & 22.654 \\
XZY & 0.81 & 19.043 \\
TS & 1.12 & 13.865 \\
ZB & 1.33 & 9.996 \\
ZC & 1.72 & 16.086 \\
YW & 2.07 & 25.334 \\
NY & 4.25 & 23.015 \\
\hline
\end{tabular}

(Zhao et al., 2020). To obtain the structure of pores in coal, the deviation correction is essential. The Porod's curve deviation correction for sample (GS sample as an example) was shown in Figure 2. The corrected SAXS data was used for subsequent processing and analysis.

The porosity of each sample was quantified by using the absolute scattering intensity. In this paper, the X-ray beam size was $1.4 \times 0.2 \mathrm{~mm}^{2}$. The thickness of the sample is $1 \mathrm{~mm}$. Combined with the sample volume and mass in the beam testing area, the porosity was then normalized to mass weighted pore volume for each sample, as presented in Table 4. Variation in pore volume of samples with coal rank is shown in Figure 3.

With changes in the metamorphic degrees of coals, the overall pore volume of coal first increased, then decreased, then increased and decreased as shown in Figure 3. The pore volume of sample GS (lignite) with the lowest degree of metamorphism is $17.587 \mathrm{~mm}^{3} / \mathrm{g}$, and that of sample SGT (bituminous C) is $22.654 \mathrm{~mm}^{3} / \mathrm{g}$. A decreasing trend in the pore volume is observed from sample SGT (bituminous C) to sample ZB (bituminous B). The pore volume of sample $\mathrm{ZB}$ (bituminous B) is $9.996 \mathrm{~mm}^{3} / \mathrm{g}$, the smallest pore volume among the eight coal samples. For coals with higher metamorphism degree, the pore volume of sample YW (bituminous A) is $25.334 \mathrm{~mm}^{3} / \mathrm{g}$, which is the largest pore volume among the eight coal samples. For sample NY (anthracite), the pore volume is slightly lower at $23.015 \mathrm{~mm}^{3} / \mathrm{g}$.

Coal is considered to be a two-phase polydisperse system composed of coal matrix and pores (Radlinski et al., 2004; Bahadur et al., 2015). In this study, the international software McSAS was used to investigate the pore distribution characteristics of each coal sample (Bressler et al., 2015). The SAXS data was analyzed using polydisperse sphere model, and the pore size distribution obtained was just a relative volume fraction. By combining this result with the pore volume per unit mass for each sample, the pore size distribution normalized to the mass weighted pore volume was obtained. The pore size distributions of various samples are presented in Figure 4. Within the range of 2-65 nm, all coal samples exhibit pore size distribution, but there are distribution differences among them. For pores with diameter less than $20 \mathrm{~nm}$, samples SGT (bituminous C), XZY (bituminous C), YW (bituminous A), NY (anthracite) have larger pore volume, indicating that these four coal samples have more pores within this pore size range. For pores with the diameter larger than $20 \mathrm{~nm}$, except for sample ZB (bituminous B), the pore size distributions of the other coal samples have no obvious

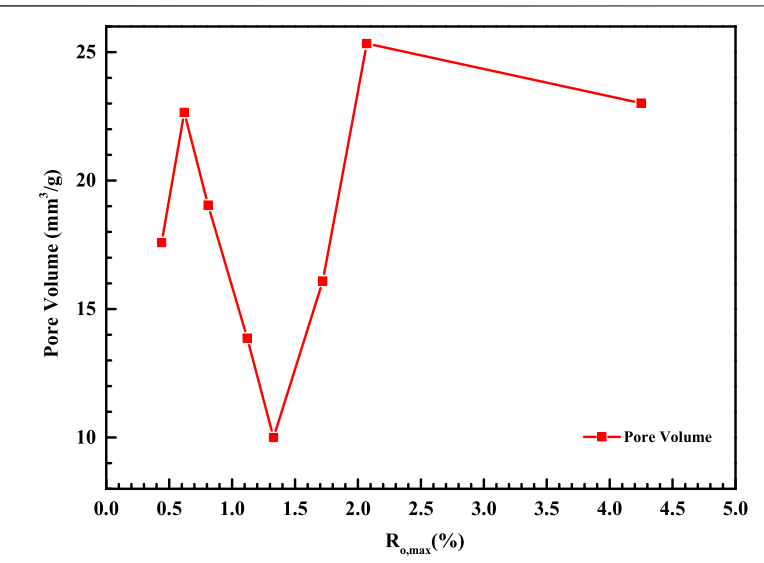

FIGURE 3 | Variation of pore volume of coal samples with coal rank.

difference. The pore volume of sample $\mathrm{ZB}$ (bituminous B) is small over the entire pore size distribution range, indicating that $\mathrm{ZB}$ (bituminous $B$ ) has few nanopores within the range of 2-65 $\mathrm{nm}$.

\section{Low-Temperature Nitrogen Adsorption Results}

The physisorption isotherms were classified into eight types by IUPAC (Thommes et al., 2015). The low-temperature $\mathrm{N}_{2}$ adsorption isotherms of the eight coals with different ranks are presented in Figure 5. The isotherms of samples are closer to a combination of type II and type IV(a) isotherms, indicating that coals have complex pore structure. On the whole, the adsorption process for coal samples can be divided into micropore filling, monolayer adsorption, multilayer adsorption and capillary condensation (Choi et al., 2001; Sing and Williams, 2005). At extremely low relative pressures $\left(P / P_{0}<0.1\right)$, the nitrogen quantity adsorbed increases obviously because nitrogen molecules fill micropores with pore size close to the diameter of gas molecules. Physisorption in mesopores and macropores is distinct from that in micropores. With increasing relative pressure, nitrogen molecules are gradually adsorbed onto surfaces of mesopores in coals, which is monolayer adsorption. Then, the adsorption isotherm is relatively flat $\left(\mathrm{P} / \mathrm{P}_{0}<0.4\right)$. In this process, multi-layer nitrogen molecules are adsorbed on pore surfaces in coals. When the relative pressure exceeds 0.4 , with continuous multilayer adsorption, an increasing number of nitrogen molecules undergo capillary condensation in the pores of coal (Hou et al., 2017). The nitrogen condenses to a liquid-like phase, and the amount of nitrogen adsorbed increases obviously.

Figure 5 shows that the types of hysteresis loops of physisorption isotherms for the eight coal samples are not consistent. Among them, the adsorption and desorption curves of some coal samples are close to overlapping, while physisorption isotherms of some coal samples have obvious hysteresis loops. Based on the latest classification of hysteresis loops from IUPAC (Thommes et al., 2015), the hysteresis loops for the eight coal samples can be divided into three types. The 

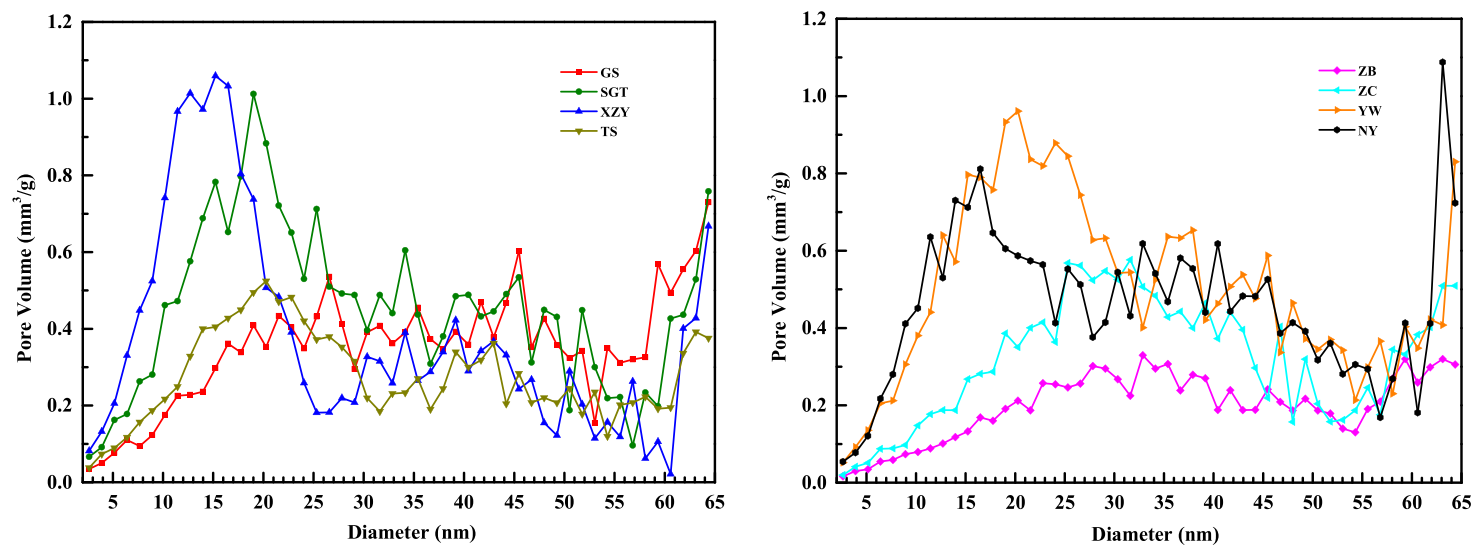

FIGURE 4 | Pore size distribution of coals with different ranks.
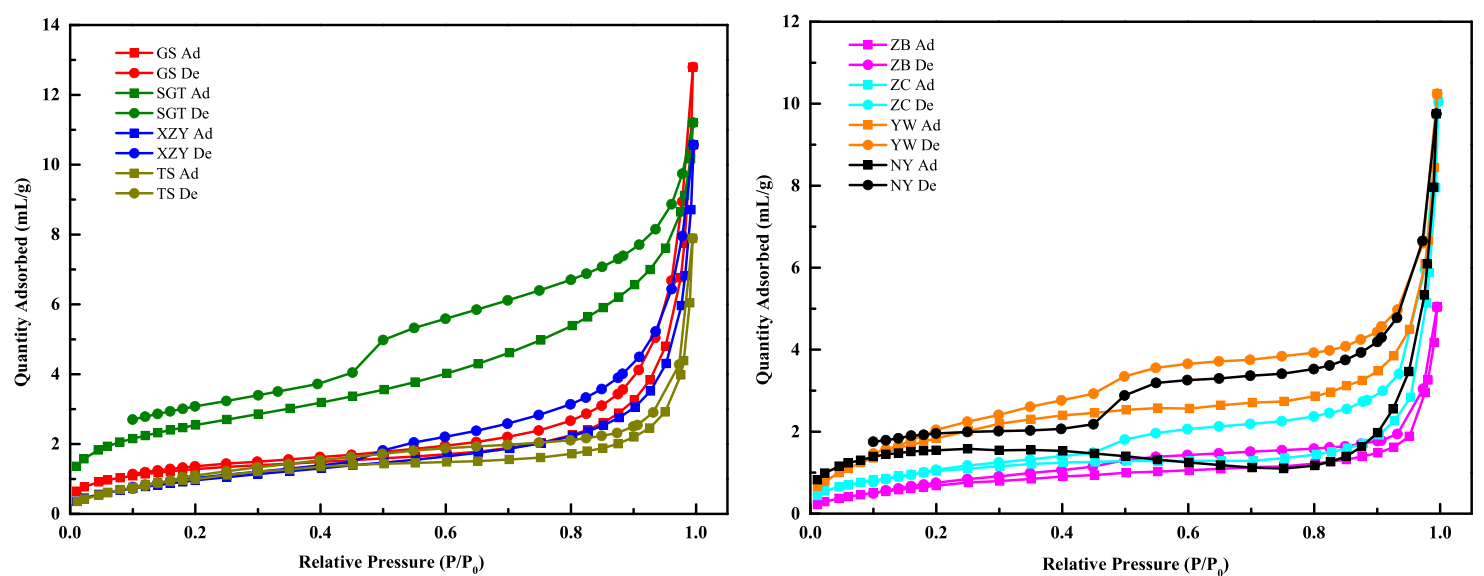

FIGURE 5 | Low temperature $\mathrm{N}_{2}$ adsorption isotherms of coal samples.
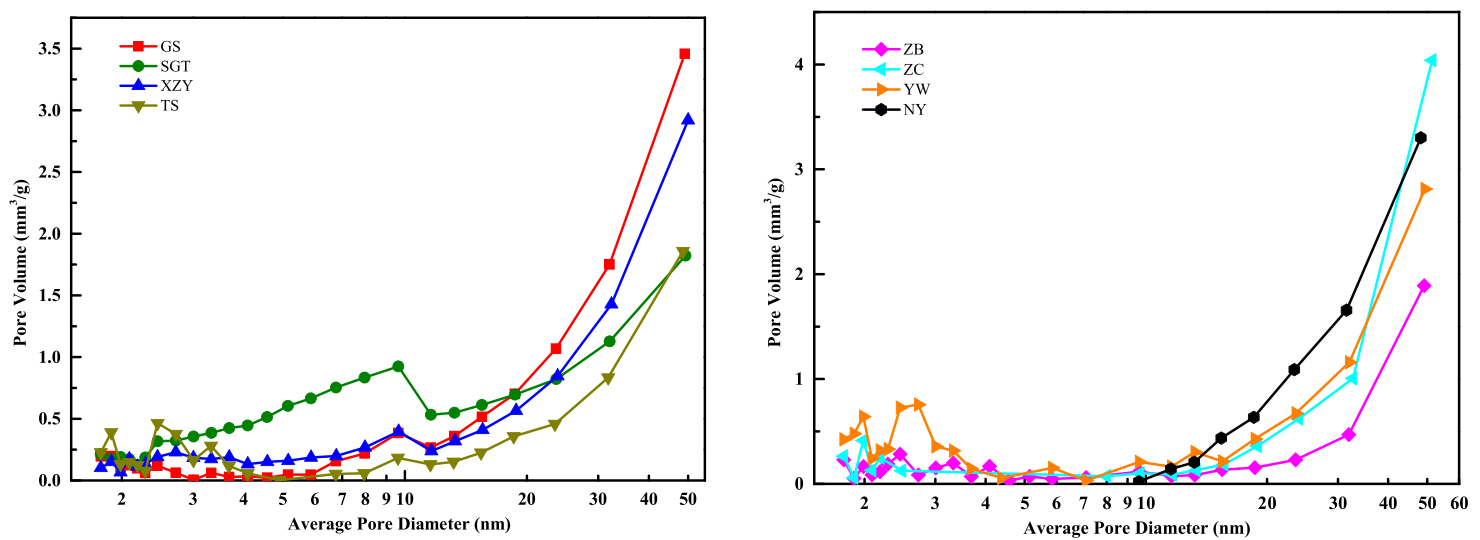

FIGURE 6 | Pore size distribution of coal samples obtained by BJH theory. 
TABLE 5 | Pore volume, open/closed porosity obtained from LTNA and SAXS experiments.

\begin{tabular}{|c|c|c|c|c|}
\hline \multirow[t]{2}{*}{ Sample ID } & \multicolumn{2}{|c|}{ Pore volume $\left(\mathrm{mm}^{3} / \mathrm{g}\right)$} & \multicolumn{2}{|c|}{ Porosity (\%) } \\
\hline & LPNA & SAXS & Open & Closed \\
\hline GS & 10.096 & 17.587 & 57.41 & 42.59 \\
\hline SGT & 13.776 & 22.654 & 60.81 & 39.19 \\
\hline$X Z Y$ & 9.927 & 19.043 & 52.13 & 47.87 \\
\hline TS & 6.869 & 13.865 & 49.54 & 50.46 \\
\hline ZB & 5.156 & 9.996 & 51.58 & 48.42 \\
\hline ZC & 7.846 & 16.086 & 48.78 & 51.22 \\
\hline YW & 10.936 & 25.334 & 43.17 & 56.83 \\
\hline NY & 7.489 & 23.015 & 32.54 & 67.46 \\
\hline
\end{tabular}

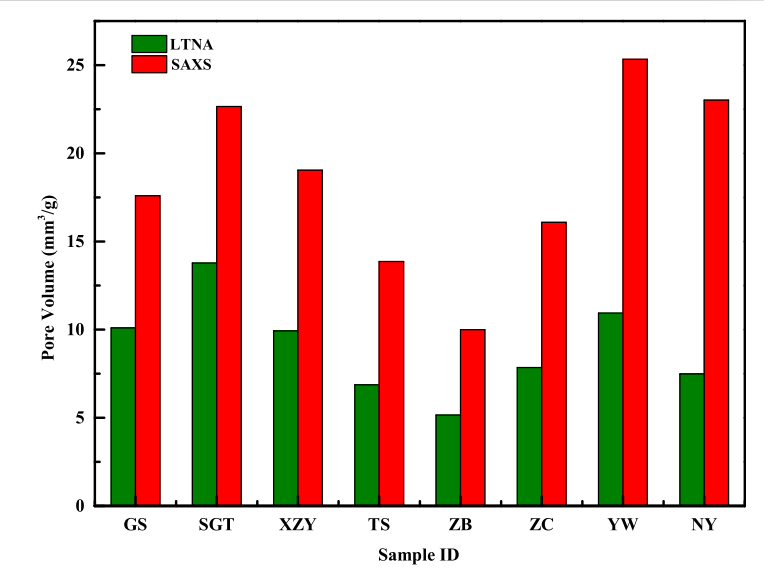

FIGURE 7 | Histogram of pore volume of coal samples obtained from LTNA and SAXS experiments.

hysteresis loops of sample GS (lignite) and sample TS (bituminous B) belong to the same type, and the hysteresis loops are inconspicuous, which indicates that their pore morphology is mainly slit-like and plate-like with one side closed (Zhao et al., 2016). The isotherms of samples XZY (bituminous A), ZB (bituminous B), ZC (bituminous C), and YW (bituminous C) show small adsorption hysteresis loops, corresponding to type $\mathrm{H} 4$. This means that the pores in these coals are mainly cylinder-like and cone-like, and they are open on both sides with good connectivity (Chen et al., 2017). The physisorption isotherms of samples SGT (bituminous A) and NY (anthracite) have obvious hysteresis loops, corresponding to type H2. Their desorption curves drop sharply when the relative pressure is less than 0.5 , demonstrating that both coals have "inkbottle" pores (He et al., 2020).

The pore size distribution can be obtained for each sample using the nitrogen adsorption curves of samples and $\mathrm{BJH}$ theory (as shown in Figure 6). Samples other than NY exhibit pores with diameter less than $10 \mathrm{~nm}$, and these contributed various pore volume. In the range of pore diameter less than $10 \mathrm{~nm}$, samples GS, SGT and XZY show the largest pore volume for the pores with approximately $10 \mathrm{~nm}$ diameter, while samples TS, ZB, ZC and YW show the largest pore volume for pores with diameter of

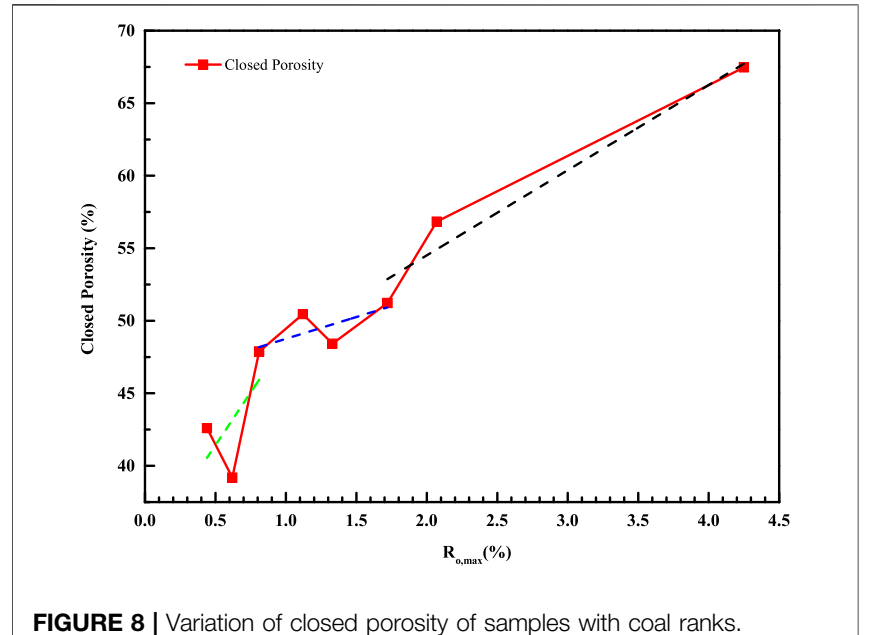

FIGURE 8 | Variation of closed porosity of samples with coal ranks.

approximately $2 \mathrm{~nm}$. Sample NY has few pores with diameter less than $10 \mathrm{~nm}$. For pores with diameter greater than $10 \mathrm{~nm}$, the pore volume of all eight coal samples increases with an increase in pore diameter.

\section{Closed Pore Characteristics and Evolution Mechanism for Coal With Different Ranks}

Using BJH theory, the pore volume was obtained for pores with diameter less than $65 \mathrm{~nm}$ in coal samples with different ranks, and this is considered to be the open pore volume of coal. By combining the LTNA experimental results with the previous SAXS results, the characteristics of open and closed pores within the range of $2-65 \mathrm{~nm}$ can be determined for each sample. The results of pore volume and open/closed porosity measured by the two kinds of experiments are shown in Table 5.

To compare the pore volume determined by LTNA and SAXS experiments for coals with different ranks, two experimental results of each coal are compared by histogram, as shown in Figure 7. According to the SAXS results, the pore volume of samples decreases in the order $\mathrm{YW}>\mathrm{NY}>\mathrm{SGT}>\mathrm{XZY}>\mathrm{GS}>$ $\mathrm{ZC}>\mathrm{TS}>\mathrm{ZB}$. According to the LTNA results, the order decreases as $\mathrm{SGT}>\mathrm{YW}>\mathrm{GS}>\mathrm{XZY}>\mathrm{ZC}>\mathrm{NY}>\mathrm{TS}>\mathrm{ZB}$. The orders of pore volume differ for the two experiments. The SAXS results show that the pore volume of YW is the largest, but the pore volume of SGT is the largest in the LTNA experiment. Moreover, the pore volume of NY is the second among the eight coal samples according to SAXS results, while its pore volume ranks sixth among the eight coal samples based on LTNA results. The difference in the orders of pore volume for the two experimental techniques reveals the difference in open and closed porosity in coals with different ranks.

In the LTNA experiments, open pores in the target range can be detected by nitrogen adsorption, while total pores information (including open pores and closed pores) in the target range can be measured by SAXS experiment. A combination of the two experimental methods effectively detects the open porosity and closed porosity within the target range for the sample, thereby evaluating the characteristics of open pores and closed pores for 
sample. The results indicate that the open porosity of sample SGT is the highest at $60.81 \%$ and that of GS is also high at $57.41 \%$, while the open porosity of medium-metamorphic coals (samples $\mathrm{XZY}, \mathrm{TS}, \mathrm{ZB}, \mathrm{ZC}$ ) is approximately $50 \%$. The open porosity of sample YW is $43.17 \%$ and that of NY is the lowest among the eight samples at $32.54 \%$. On the whole, the open porosity of lowmetamorphic coals is relatively higher, the open porosity of medium-metamorphic coals is in the middle, and the open porosity of high-metamorphic coals is relatively lower. Accordingly, the closed porosity of coals shows the opposite trend.

Figure 8 shows the variation in closed porosity of samples with coal ranks. The figure was divided into three parts: lowmetamorphic coals (GS and SGT); medium-metamorphic coals (XZY, TS, ZB, ZC) and high-metamorphic coals (YW and NY). Fitting the closed porosity of the three parts basically shows that the closed porosity increases from low-metamorphic coals to medium-metamorphic coals, the closed porosity of mediummetamorphic coals increases slightly with the increase of coal rank, and the closed porosity of high-metamorphic coal increases significantly with the increase of coal rank. This may be related to the different molecular structure of coals with different ranks (Liu et al., 2019; Ren et al., 2021).

For low-metamorphic coals, the molecules contain more irregular parts, such as aliphatic chains and functional groups, while there are few aromatic rings (Yan et al., 2020a). The gaps between them often form large pores, and the macroscopic structure of coals is loose (Jiang et al., 2019b). Because there are a great number of chain structures in lowmetamorphic coals, their overall molecular structure arrangement is disordered and spaced. It is easier for side chains, bridge bonds and functional groups to form connected pores with each other. Therefore, there are many connected pores in low-metamorphic coals, and the extent of their closed porosity is low. For medium-metamorphic coals, most of the alkyl side chains and functional groups have fallen off (Yan et al., 2020b). At the same time, aromatization has led to more aromatic rings structure and the intermolecular arrangement has become relatively dense (Jiang et al., 2019a). Due to the losses of side chains and functional groups, aromatic rings are typically connected by bridge bonds, which reduces the opportunity to form connected pores. In addition, aromatization converts the aliphatic chain structures into closed aromatic rings, which may also reduce the open porosity and increase the closed porosity of mediummetamorphic coals. For high-metamorphic coals, the mechanism for molecular evolution is mainly polycondensation, and irregular parts such as functional groups and side chains have basically disappeared (Meng et al., 2020). The degree of condensation for aromatic rings in coal macromolecules has improved continuously. The aromatic units extend horizontally and stack vertically, which obviously improves the order and density of the corresponding macromolecular structure (Niu et al., 2019). The dense arrangement of the molecular structure provides for easy formation of closed pores between aromatic rings in high-metamorphic coals. Therefore, the closed porosity in high-metamorphic coal is significantly increased.

Previous studies have shown that when selecting powdered samples for LTNA experiments, the number of open pores in coal is increased during the pulverization process (Jin et al., 2016; Si et al., 2017). The quantity of nitrogen adsorbed by samples with small particle sizes in the LTNA experiment is greater, and the corresponding open pore volume is larger. Our previous research shows that pulverization also increases the total pores (both open and closed pores) volume in coal (Zhao et al., 2020). In this paper, slice samples similar to the original state of coal were selected for SAXS experiment, and 60-80 mesh particle samples were selected for LTNA experiment. This may increase the open pore volume obtained from LTNA experiment relative to that of the original sample. Therefore, the open porosity values obtained in this study may be larger than the open porosity present in the original state of coal, and the corresponding closed porosity may be smaller. The actual closed porosity in coal may be greater than those determined experimentally in this paper.

\section{CONCLUSION}

To study the characteristics of open and closed pores in coals with different ranks, the SAXS and LTNA experiments were used for joint analysis in this work. The characteristics of open and closed pores with size in the range of 2-65 $\mathrm{nm}$ for various coals were measured, and the mechanism for evolution of open and closed pores during coalification was analyzed for coals with different ranks. The main conclusions are as follows:

1) The orders of pore volume sizes of the SAXS experimental results and the LTNA experimental results are not consistent. The difference in orders of pore volume reveals the difference in open and closed porosity in coals with different ranks.

2) Overall, the closed porosity of low-metamorphic coals is relatively lower, the closed porosity of mediummetamorphic coals is in the middle, and the closed porosity of high-metamorphic coals is relatively higher. Accordingly, the open porosity of coals shows the opposite trend.

3) The change in closed porosity for coals with different ranks may be related to differences in coal molecular structure. The low-metamorphic coals have more disordered arrangement of molecular structure and easily form connected pores. Therefore, the closed porosity in low-metamorphic coals is low. The aromatization of medium-metamorphic coals turns aliphatic chain structures into closed aromatic rings, and the closed porosity of the corresponding coals also increases. When coals reach a high degree of metamorphism, polycondensation compacts the coal macromolecular structure, providing for easy formation of closed pores between aromatic condensed rings, so the closed porosity is obviously increased in highmetamorphic coals. 


\section{DATA AVAILABILITY STATEMENT}

The raw data supporting the conclusion of this article will be made available by the authors, without undue reservation.

\section{AUTHOR CONTRIBUTIONS}

TL, Testing, Data curation, Writing-original draft. YZ, Conceptualization, Methodology, Reviewing and Editing. NN, Reviewing and Editing.

\section{FUNDING}

This research received financial support from the National Natural Science Foundation of China (Nos. U1910206,

\section{REFERENCES}

Alexeev, A. D., Vasilenko, T. A., and Ulyanova, E. V. (1999). Closed Porosity in Fossil Coals. Fuel 78, 635-638. doi:10.1016/S0016-2361(98)00198-7

Bahadur, J., Radlinski, A. P., Melnichenko, Y. B., Mastalerz, M., and Schimmelmann, A. (2015). Small-Angle and Ultrasmall-Angle Neutron Scattering (SANS/USANS) Study of New Albany Shale: A Treatise on Microporosity. Energy Fuels 29, 567-576. doi:10.1021/ ef502211w

Bressler, I., Pauw, B. R., and Thünemann, A. F. (2015). McSAS: Software for the Retrieval of Model Parameter Distributions from Scattering Patterns. J. Appl. Cryst. 48, 962-969. doi:10.1107/S1600576715007347

Bustin, R. M., and Clarkson, C. R. (1998). Geological Controls on Coalbed Methane Reservoir Capacity and Gas Content. Int. J. Coal Geol. 38, 3-26. doi:10.1016/ S0166-5162(98)00030-5

Chen, S., Tao, S., Tang, D., Xu, H., Li, S., Zhao, J., et al. (2017). Pore Structure Characterization of Different Rank Coals Using $\mathrm{N}_{2}$ and $\mathrm{CO}_{2}$ Adsorption and its Effect on $\mathrm{CH}_{4}$ Adsorption Capacity: A Case in Panguan Syncline, Western Guizhou, China. Energy Fuels 31, 6034-6044. doi:10.1021/ acs.energyfuels.7b00675

Chen, Y., Qin, Y., Luo, Z., Yi, T., Wei, C., Wu, C., et al. (2019). Compositional Shift of Residual Gas during Desorption from Anthracite and its Influencing Factors. Fuel 250, 65-78. doi:10.1016/j.fuel.2019.03.144

Chen, Y., Qin, Y., Wei, C., Huang, L., Shi, Q., Wu, C., et al. (2018). Porosity Changes in Progressively Pulverized Anthracite Subsamples: Implications for the Study of Closed Pore Distribution in Coals. Fuel 225, 612-622. doi:10.1016/ j.fuel.2018.03.164

Choi, J.-G., Do, D. D., and Do, H. D. (2001). Surface Diffusion of Adsorbed Molecules in Porous Media: Monolayer, Multilayer, and Capillary Condensation Regimes. Ind. Eng. Chem. Res. 40, 4005-4031. doi:10.1021/ ie $010195 z$

Dai, S., Bechtel, A., Eble, C. F., Flores, R. M., French, D., Graham, I. T., et al. (2020). Recognition of Peat Depositional Environments in Coal: A Review. Int. J. Coal Geol. 219, 103383. doi:10.1016/j.coal.2019.103383

Flores, R. M. (1998). Coalbed Methane: from hazard to Resource. Int. J. Coal Geol. 35, 3-26. doi:10.1016/S0166-5162(97)00043-8

Han, W., Zhou, G., Gao, D., Zhang, Z., Wei, Z., Wang, H., et al. (2020). Experimental Analysis of the Pore Structure and Fractal Characteristics of Different Metamorphic Coal Based on Mercury Intrusion-nitrogen A-dsorption Porosimetry. Powder Technol. 362, 386-398. doi:10.1016/ j.powtec.2019.11.092

He, X., Cheng, Y., Hu, B., Wang, Z., Wang, C., Yi, M., et al. (2020). Effects of Coal Pore Structure on Methane-Coal Sorption Hysteresis: An Experimental Investigation Based on Fractal Analysis and Hysteresis Evaluation. Fuel 269, 117438. doi:10.1016/j.fuel.2020.117438
51861145403, 51874312), Science and Technology Plant Project of Inner Mongolia Autonomous Region (No. 2019GG140), Major Scientific and Technological Innovation Project of Shandong Province (Nos. 2019SDZY01, 2019SDZY02), Fundamental Research Funds for the Central Universities and Fund of China Scholarship Council.

\section{ACKNOWLEDGMENTS}

The authors are grateful to Zhihong Li and Guang Mo of Beijing Synchrotron Radiation Facility (BSRF) for providing the SAXS experimental facilities and their recommendations on SAXS experiments. We thank Yong $\mathrm{Li}$ of China University of Mining and Technology (Beijing) for his help in the lowtemperature nitrogen adsorption experiment.

Hou, S., Wang, X., Wang, X., Yuan, Y., Pan, S., and Wang, X. (2017). Pore Structure Characterization of Low Volatile Bituminous Coals with Different Particle Size and Tectonic Deformation Using Low Pressure Gas Adsorption. Int. J. Coal Geol. 183, 1-13. doi:10.1016/j.coal.2017.09.013

Hu, B., Cheng, Y., He, X., Wang, Z., Jiang, Z., Wang, C., et al. (2020). New Insights into the $\mathrm{CH}_{4}$ Adsorption Capacity of Coal Based on Microscopic Pore Properties. Fuel 262, 116675. doi:10.1016/j.fuel.2019.116675

Jiang, J., Yang, W., Cheng, Y., Liu, Z., Zhang, Q., and Zhao, K. (2019a). Molecular Structure Characterization of Middle-High Rank Coal via XRD, Raman and FTIR Spectroscopy: Implications for Coalification. Fuel 239, 559-572. doi:10.1016/j.fuel.2018.11.057

Jiang, J., Yang, W., Cheng, Y., Zhao, K., and Zheng, S. (2019b). Pore Structure Characterization of Coal Particles via MIP, $\mathrm{N}_{2}$ and $\mathrm{CO}_{2}$ Adsorption: Effect of Coalification on Nanopores Evolution. Powder Technol. 354, 136-148. doi:10.1016/j.powtec.2019.05.080

Jin, K., Cheng, Y., Liu, Q., Zhao, W., Wang, L., Wang, F., et al. (2016). Experimental Investigation of Pore Structure Damage in Pulverized Coal: Implications for Methane Adsorption and Diffusion Characteristics. Energy Fuels 30, 10383-10395. doi:10.1021/acs.energyfuels.6b02530

Levine, J. R. (1993). "Coalification: The Evolution of Coal as Source Rock and Reservoir Rock for Oil and Gas," in Hydrocarbons From Coal. Editors Law, B. E., and Rice, D. D. (Tulsa, OK: American Association of Petroleum Geologists), 39-77. doi:10.1306/St38577C3

Li, J., Jiao, A., Chen, S., Wu, Z., Xu, E., and Jin, Z. (2018). Application of the SmallAngle X-ray Scattering Technique for Structural Analysis Studies: A Review. J. Mol. Struct. 1165, 391-400. doi:10.1016/j.molstruc.2017.12.031

Li, P., Zhou, S., Zhang, X., Li, J., Zhang, S., Hou, A., et al. (2020). Analysis on Correlation between Nanopores and Coal Compositions during thermal Maturation Process. Mar. Pet. Geol. 121, 104608. doi:10.1016/ j.marpetgeo.2020.104608

Li, Z.-H. (2013). A Program for SAXS Data Processing and Analysis. Chin. Phys. C 37, 108002. doi:10.1088/1674-1137/37/10/108002

Li, Z., Liu, D., Cai, Y., Ranjith, P. G., and Yao, Y. (2017). Multi-scale Quantitative Characterization of 3-D Pore-Fracture Networks in Bituminous and Anthracite Coals Using FIB-SEM Tomography and X-Ray $\mu$-CT. Fuel 209, 43-53. doi:10.1016/j.fuel.2017.07.088

Li, Z., Liu, D., Cai, Y., Wang, Y., and Teng, J. (2019). Adsorption Pore Structure and its Fractal Characteristics of Coals by $\mathrm{N}_{2}$ Adsorption/desorption and FESEM Image Analyses. Fuel 257, 116031. doi:10.1016/j.fuel.2019.116031

Li, Z., Wu, Z., Mo, G., Xing, X., and Liu, P. (2014). A Small-Angle X-ray Scattering Station at Beijing Synchrotron Radiation Facility. Instrum. Sci. Technol. 42, 128-141. doi:10.1080/10739149.2013.845845

Liu, Y., Zhu, Y., and Chen, S. (2019). Effects of Chemical Composition, Disorder Degree and Crystallite Structure of Coal Macromolecule on Nanopores (0.4-150 $\mathrm{Nm}$ ) in Different Rank Naturally-Matured Coals. Fuel 242, 553-561. doi:10.1016/j.fuel.2019.01.095 
Liu, Y., Zhu, Y., Liu, S., Chen, S., Li, W., and Wang, Y. (2018). Molecular Structure Controls on Micropore Evolution in Coal Vitrinite during Coalification. Int. J. Coal Geol. 199, 19-30. doi:10.1016/j.coal.2018.09.012

Meng, J., Niu, J., Meng, H., Xia, J., and Zhong, R. (2020). Insight on Adsorption Mechanism of Coal Molecules at Different Ranks. Fuel 267, 117234. doi:10.1016/j.fuel.2020.117234

Mou, P., Pan, J., Niu, Q., Wang, Z., Li, Y., and Song, D. (2021). Coal Pores: Methods, Types, and Characteristics. Energy Fuels 35, 7467-7484. doi:10.1021/ acs.energyfuels.1c00344

Nie, B., Liu, X., Yang, L., Meng, J., and Li, X. (2015). Pore Structure Characterization of Different Rank Coals Using Gas Adsorption and Scanning Electron Microscopy. Fuel 158, 908-917. doi:10.1016/ j.fuel.2015.06.050

Niu, Q., Pan, J., Cao, L., Ji, Z., Wang, H., Wang, K., et al. (2017). The Evolution and Formation Mechanisms of Closed Pores in Coal. Fuel 200, 555-563. doi:10.1016/j.fuel.2017.03.084

Niu, Q., Pan, J., Jin, Y., Wang, H., Li, M., Ji, Z., et al. (2019). Fractal Study of Adsorption-Pores in Pulverized Coals with Various Metamorphism Degrees Using $\mathrm{N}_{2}$ Adsorption, X-ray Scattering and Image Analysis Methods. J. Pet. Sci. Eng. 176, 584-593. doi:10.1016/j.petrol.2019.01.107

Okolo, G. N., Everson, R. C., Neomagus, H. W. J. P., Roberts, M. J., and Sakurovs, R. (2015). Comparing the Porosity and Surface Areas of Coal as Measured by Gas Adsorption, Mercury Intrusion and SAXS Techniques. Fuel 141, 293-304. doi:10.1016/j.fuel.2014.10.046

Pan, J., Niu, Q., Wang, K., Shi, X., and Li, M. (2016). The Closed Pores of Tectonically Deformed Coal Studied by Small-Angle X-ray Scattering and Liquid Nitrogen Adsorption. Micropor. Mesopor. Mater. 224, 245-252. doi:10.1016/j.micromeso.2015.11.057

Radlinski, A. P., Mastalerz, M., Hinde, A. L., Hainbuchner, M., Rauch, H., Baron, M., et al. (2004). Application of SAXS and SANS in Evaluation of Porosity, Pore Size Distribution and Surface Area of Coal. Int. J. Coal Geol. 59, 245-271. doi:10.1016/j.coal.2004.03.002

Radlinski, A. P. (2006). Small-angle Neutron Scattering and the Microstructure of Rocks. Rev. Mineralogy Geochem. 63, 363-397. doi:10.2138/rmg.2006.63.14

Ren, J., Song, Z., Li, B., Liu, J., Lv, R., and Liu, G. (2021). Structure Feature and Evolution Mechanism of Pores in Different Metamorphism and Deformation Coals. Fuel 283, 119292. doi:10.1016/j.fuel.2020.119292

Si, L., Li, Z., Yang, Y., Xin, L., Liu, Z., Liu, Y., et al. (2017). Experimental Investigation for Pore Structure and $\mathrm{CH}_{4}$ Release Characteristics of Coal during Pulverization Process. Energy Fuels 31, 14357-14366. doi:10.1021/ acs.energyfuels.7b01995

Sing, K. S. W., and Williams, R. T. (2005). Empirical Procedures for the Analysis of Physisorption Isotherms. Adsorpt. Sci. Technol. 23, 839-853. doi:10.1260/ 026361705777641990

Thommes, M., Kaneko, K., Neimark, A. V., Olivier, J. P., Rodriguez-Reinoso, F., Rouquerol, J., et al. (2015). Physisorption of Gases, with Special Reference to the Evaluation of Surface Area and Pore Size Distribution (IUPAC Technical Report). Pure Appl. Chem. 87, 1051-1069. doi:10.1515/pac-2014-1117

Wang, A., Cao, D., Wei, Y., Nie, J., and Qin, R. (2020). Comparison of Nanopore Evolution in Vitrinite and Inertinite in Coalbed Methane Reservoirs during Coalification. J. Nat. Gas Sci. Eng. 78, 103289. doi:10.1016/j.jngse.2020.103289
Wang, F., Liang, Y., and Zou, Q. (2019). Correlation Between Coal and Gas Outburst Risk and Adsorption Properties of Coal Seams. Energy Sci. Eng. 7, 974-985. doi:10.1002/ese3.326

Wang, Z., Wang, H., Yang, Y., Deng, Z., Fu, X., Pan, J., et al. (2021). Effect of the Coal Molecular Structure on the Micropore Volume and the Coalbed Methane Content. Energy Fuels 35, 19437-19447. doi:10.1021/acs.energyfuels.1c02889

Xie, F., Li, Z., Li, Z., Li, D., Gao, Y., and Wang, B. (2018). Absolute Intensity Calibration and Application at BSRF SAXS Station. Nucl. Instrum. Methods Phys. Res. Sect. A Accel. Spectrom. Detect. Assoc. Equip. 900, 64-68. doi:10.1016/ j.nima.2018.05.026

Yan, J., Lei, Z., Li, Z., Wang, Z., Ren, S., Kang, S., et al. (2020a). Molecular Structure Characterization of Low-Medium Rank Coals via XRD, Solid State ${ }^{13} \mathrm{C}$ NMR and FTIR Spectroscopy. Fuel 268, 117038. doi:10.1016/ j.fuel.2020.117038

Yan, J., Meng, Z., Zhang, K., Yao, H., and Hao, H. (2020b). Pore Distribution Characteristics of Various Rank Coals Matrix and Their Influences on Gas Adsorption. J. Pet. Sci. Eng. 189, 107041. doi:10.1016/j.petrol.2020.107041

Yao, Y., Liu, D., Tang, D., Tang, S., and Huang, W. (2008). Fractal Characterization of Adsorption-Pores of Coals from North China: An Investigation on $\mathrm{CH}_{4}$ Adsorption Capacity of Coals. Int. J. Coal Geol. 73, 27-42. doi:10.1016/ j.coal.2007.07.003

Zhao, J., Xu, H., Tang, D., Mathews, J. P., Li, S., and Tao, S. (2016). A Comparative Evaluation of Coal Specific Surface Area by $\mathrm{CO}_{2}$ and $\mathrm{N}_{2}$ Adsorption and its Influence on $\mathrm{CH}_{4}$ Adsorption Capacity at Different Pore Sizes. Fuel 183, 420-431. doi:10.1016/j.fuel.2016.06.076

Zhao, Y., Liu, S., Elsworth, D., Jiang, Y., and Zhu, J. (2014). Pore Structure Characterization of Coal by Synchrotron Small-Angle X-ray Scattering and Transmission Electron Microscopy. Energy Fuels 28, 3704-3711. doi:10.1021/ ef500487d

Zhao, Y., Liu, T., Danesh, N. N., Sun, Y., Liu, S., and Wang, Y. (2020). Quantification of Pore Modification in Coals Due to Pulverization Using Synchrotron Small Angle X-ray Scattering. J. Nat. Gas Sci. Eng. 84, 103669. doi:10.1016/j.jngse.2020.103669

Conflict of Interest: The authors declare that the research was conducted in the absence of any commercial or financial relationships that could be construed as a potential conflict of interest.

Publisher's Note: All claims expressed in this article are solely those of the authors and do not necessarily represent those of their affiliated organizations, or those of the publisher, the editors and the reviewers. Any product that may be evaluated in this article, or claim that may be made by its manufacturer, is not guaranteed or endorsed by the publisher.

Copyright (C) $2021 \mathrm{Liu}$, Zhao and Noraei Danesh. This is an open-access article distributed under the terms of the Creative Commons Attribution License (CC BY). The use, distribution or reproduction in other forums is permitted, provided the original author(s) and the copyright owner(s) are credited and that the original publication in this journal is cited, in accordance with accepted academic practice. No use, distribution or reproduction is permitted which does not comply with these terms. 